\title{
Uso de la Metacognición como factor potencializador del aprendizaje
}

\section{Use of Metacognition as a learning potential factor}

\author{
ARZOLA-MONREAL, Juan Martin†, FARIAS-BRACAMONTES, Juan Carlos*, GARCÍA-GARCÍA, \\ Jesús Alberto y CUFARFÁN-LÓPEZ, Julio
}

Universidad Autónoma de Coahuila-Facultad de Ciencia, Educación y Humanidades

ID $1^{\text {er }}$ Autor: Juan Martin, Arzola-Monreal

ID $1^{\text {er }}$ Coautor: Juan Carlos, Farías-Bracamontes / ORC ID: 0000-0003-0939-3608, CVU CONACYT ID: 371500

ID $2^{\text {do }}$ Coautor: Jesús Alberto, García-García / ORC ID: 0000-0003-1369-311X, Researcher ID Thomson: D-8211-2019, CVU CONACYT ID: 568438

ID $3^{\text {er }}$ Coautor: Julio, Cufarfán-López / ORC ID: 0000-0001-7203-8022

DOI: $10.35429 /$ JCP.2020.12.4.15.20

Recibido 03 de Agosto, 2020; Aceptado 30 de Diciembre, 2020

\section{Resumen}

El aprendizaje es un eje fundamental en el desarrollo del individuo que dispone de numerosas formas de percibirse, desarrollarse e implementarse. Una búsqueda constante ha sido la proposición de formas diversas de desarrollar en el individuo este concepto, sobre todo el de tipo lógico-matemático; el que refiere a su potencialización, es una parte medular donde a través del análisis de procesos metacognitivos y estructuras curriculares complementarias, fue posible la obtención de conocimiento analizado y utilizado de una forma más provechosa en el individuo. Este estudio se enfocó a población universitaria y se utilizó para la obtención de datos estadísticos, un instrumento que abordó los ejes de aprendizaje, metacognición y currículo. De los resultados obtenidos, se identificaron elementos de comprensión y verificación afines al aprendizaje que resultaron serlo también a la metacognición. Para la investigación se utilizó un diseño de investigación de tipo mixto debido a que su estructura, estuvo conformada por dos bloques principales para la obtención de información.

\begin{abstract}
Learning is a fundamental axis in the development of the individual who has many ways of perceiving, developing and implementing himself. A constant search has been the proposition of different ways to develop this concept in the individual, especially the logical-mathematical one; The one that refers to its potentiation, is a core part where through the analysis of metacognitive processes and complementary curricular structures, it was possible to obtain knowledge analyzed and used in a more profitable way in the individual. This study focused on the university population and was used to obtain statistical data, an instrument that addressed the axes of learning, metacognition and curriculum. From the results obtained, comprehension and verification elements related to learning were identified, which also turned out to be related to metacognition. For the research, a mixed-type research design was used because its structure consisted of two main blocks for obtaining information.
\end{abstract}

Citación: ARZOLA-MONREAL, Juan Martin, FARIAS-BRACAMONTES, Juan Carlos, GARCÍA-GARCÍA，Jesús Alberto y CUFARFÁN-LÓPEZ, Julio. Uso de la Metacognición como factor potencializador del aprendizaje. Revista de Pedagogía Crítica. 2020, 4-12: 15-20

\footnotetext{
* Correspondencia del Autor (carlos.farias@uadec.edu.mx)

$\dagger$ Investigador contribuyendo como primer autor.
} 


\section{Introducción}

La siguiente investigación involucra un estudio exhaustivo acerca de la pertinencia de la metacognición y su influencia en la forma en que beneficia al aprendizaje mediante su intervención como factor Potencializador. Si bien este concepto tiene ya tiempo desde su inclusión a finales de lo 70's, han surgido diversas formas de interpretarlo y aplicarlo en los diferentes niveles educativos y cada país considerando su "forma", pero en algunos países en vías de desarrollo, no el "fondo". $\mathrm{La}$ importancia de la metacognición en el aprendizaje, siguiendo de la mano a los diversos estudios, respaldan su valor en una fórmula compleja llamada "aprendizaje" donde se incluye la adjunción de estrategias cognitivas en el currículo, que sean en parte, desarrolladoras de conocimiento por parte de los alumnos. Es pues, la forma en que los individuos pueden lograr llegar al "aprender a aprender" donde no hay limitantes respecto al contexto donde las personas hagan uso de este aprendizaje.

Los ejes principales de este estudio son: la metacognición, el principal de ellos, potencialidad y currículo; La metacognición representa la definición clara de lo que se quiere lograr (aprender) y los recursos disponibles que se tengan para lograrlo, refiriendo lo anterior a "ser consciente" de nuestro aprendizaje y ejecutar toma de decisiones para la mejora del mismo. En el caso de la potencialidad de aprendizaje, existe una fundamentación basada en estudios y aplicaciones previas de que el desarrollo de este aspecto viene dado por aplicar componentes reflexivos orientados a la lógica matemática, donde la incorporación de razonamientos deductivos donde la realidad normal se altera o modifica bajo ciertas circunstancias ayude en esta "potencialidad" de conocimiento.

\section{Metodología de estudio}

Para la realización de esta investigación, se incluyen en el instrumento aplicado, elementos de resolución de problemas enfocados al a percepción, de tipo directo e indirecto, inductivo y deductivo, representación espacial, entre otros; se utilizaron pruebas de confiabilidad y validez, así como la inclusión de las respectivas consideraciones descriptivas y cualitativas; de las cuales se derivan frecuencias, cuartiles, varianza, sedimentación, matriz de componentes, entre otros.

ISSN 2523-2479

ECORFAN $^{\circledR}$ Todos los derechos reservados
La selección de la muestra y su aplicación correspondiente se desarrollaron en una institución de educación superior; mediante la aplicación de un instrumento previamente diseñado y validado, éste se aplicó a una muestra valida de alumnos de diversos semestres de un programa educativo de ingeniería, enfocado al área de informática y sistemas computacionales. En diversos campos analizados de pudo constatar que el término de metacognición es más bien enfocado con un enfoque tradicional y dirigido aún, a una forma de aprendizaje unidireccional por lo que aquí radica su importancia ya que, al conocer el fondo y beneficios de la aplicación de esta estrategia de reflexión, es posible que la formación.

Conforme a la información recolectada y en función de la propuesta de este proyecto de investigación, esta se basa en término de buscar la implicación que tienen los procesos característicos denotados por la metacognición como forma de integración relacionada con la potencialidad del aprendizaje, específicamente del lógico matemático; lo anterior a través del apoyo en la identificación y contraste de los actuales esquemas curriculares de los que son partícipe la mayoría de las instituciones educativas.

Inicialmente, se identificaron los componentes elementales de cada uno de los ejes constituyentes de la investigación considerando el objeto de estudio, la población seleccionada y la formulación de las hipótesis de investigación. Se identificaron las variables de estudio para la conformación del instrumento de aplicación, el cual una vez aplicado se sometió a pruebas de validez y análisis estadístico. Con la información anterior, se elaboró la propuesta y formulación de resultados correspondientes, así como las recomendaciones e implicaciones del estudio.

La aplicación de las pruebas e instrumentos de investigación intentan brindar un sendero que muestre la pertinencia de la reflexión en los sujetos para la realización de actividades complejas. Parte de la propuesta que en este proyecto se realiza, es en sí la identificación de la necesidad de reflexividad en el ser humano como punto de partida de su desarrollo personal y más específicamente académico. 
Para esta investigación se utilizó un diseño de investigación de tipo mixto debido a que su estructura, estuvo conformada por dos bloques principales para la obtención de información. Dichos bloques consistieron en investigación cualitativa integrada en el instrumento aplicado por datos como preguntas que aludieron a estados de satisfacción y por otra parte las de tipo cuantitativas ya que el instrumento considera el número de respuestas, su tipo, y la cantidad de estas conforme a reglas más generalizadas. Lo anterior se justifica porque en el proceso de obtención de información acerca de la metacognición, aprendizaje y contenidos curriculares; se identificaron elementos preponderantes en cada uno de esos ejes. En el primero, metacognición, resulta necesaria la correlación de formas abstractas en el respondiente reflejado en el instrumento, se propuso a través de una serie de reactivos que por una parte planteaban problemáticas de tipo lógico y matemático y por otra, los relacionaban con reflexiones sobre su actuar enfatizando el tipo de respuesta del sustentante.

Del otro eje, el del aprendizaje, se diseñó con el fin de identificar comportamientos que ayudarán al alumno a desarrollar su potencial de aprendizaje. En el caso del último de los ejes, este fue considerado por la estructuración de la información que al alumno se le plantea en cualquier institución de educación, y que, en algunos casos puede influir en la disminución del aprendizaje en el alumno.

De la clasificación de Kerlinger (1988), se desprende la siguiente derivación de las variables de esta investigación: Considerando el tipo de medición, se utilizaron variables nominales y ordinales mencionando que este grupo de variables se aplicó en los datos de identificación del respondiente, en tanto que, para la parte de las preguntas del instrumento, fueron utilizadas variables de tipo nominal en una escala de cero a diez, y también en la selección de respuesta única de las múltiples.

En la presentación del instrumento, fueron solicitados los siguientes datos: edad, peso, estatura, descendientes, estado civil, tipo de sangre, género, tipo de formación académica, nivel de clasificación en la actividad de sueño, alimento, deporte, tabaco y economía familiar.
Adicionalmente se solicitó el promedio global anterior inmediato y el semestre y la carrera que cursaban, remarcando en éste último que toda la población fue focalizada en uno solo, que fue el de la carrera de ingeniería en sistemas computacionales.

Respecto a la conformación de la primera de las tres secciones, en ésta se presentaron una serie de 17 reactivos de tipo dicotómico donde el sustentante, basado en un planteamiento inicial respondía a la que, según su criterio fue la respuesta correcta. Para el procesamiento de la información en esta sección se declaró en la sección de variables con un cero la respuesta incorrecta del respondiente mientras que a la respuesta correcta se le asignó un valor de uno.

Sólo se presentó un caso en el reactivo 17, donde la respuesta tuvo valor de puntaje diferente al máximo de valor de uno, lo anterior debido a que este reactivo se diseñó bajo el planteamiento del nivel de percepción, imaginación y lógica del sustentante al visualizar una figura; de este procesamiento, los niveles de respuesta fueron considerados con cero puntos si no contestó ninguna de las cuatro solicitudes de respuesta, un punto si contestó los tres de las 4 peticiones de respuesta sin considerar la más compleja, la del inciso "a", y dos puntos si se presentaba el caso anterior pero considerando que el respondiente hubiera contestado de entre sus tres respuestas la más compleja, el inciso "a". Finalmente, se le otorgaron tres puntos en el caso de que el respondiente respondiera afirmativamente todas las cuatro peticiones de respuesta

\section{Resultados}

En la parte transversal de este estudio se dispone como lo indica la Figura 1, que la metacognición es un aspecto esencial para el fomento del aprendizaje. Los componentes reflexivos sobre el pensamiento que se mencionan en el eje de metacognición son la reflexión transpuesta como crítica externa de su entorno, el análisis de ideas, la auto-percepción o introspección del individuo en las tareas que desempeña, la atención focalizada mediante la observación y la autoreflexión propia a las actividades del individuo. 
Mediante los anteriores aspectos, el aprendizaje dispone de factores que pueden ser mejorados con la combinación o fomento de las tareas metacognitivas, componentes como la lógica, ordenamiento o jerarquización de ideas, interpretación sensorial y sobre todo el de evaluación, que está estrechamente relacionado a una reflexión constante y sinérgica; este último aspecto se correlaciona porque es inherente que para una reflexión haya una evaluación y en forma viceversa se requiera de una evaluación para una reflexión.

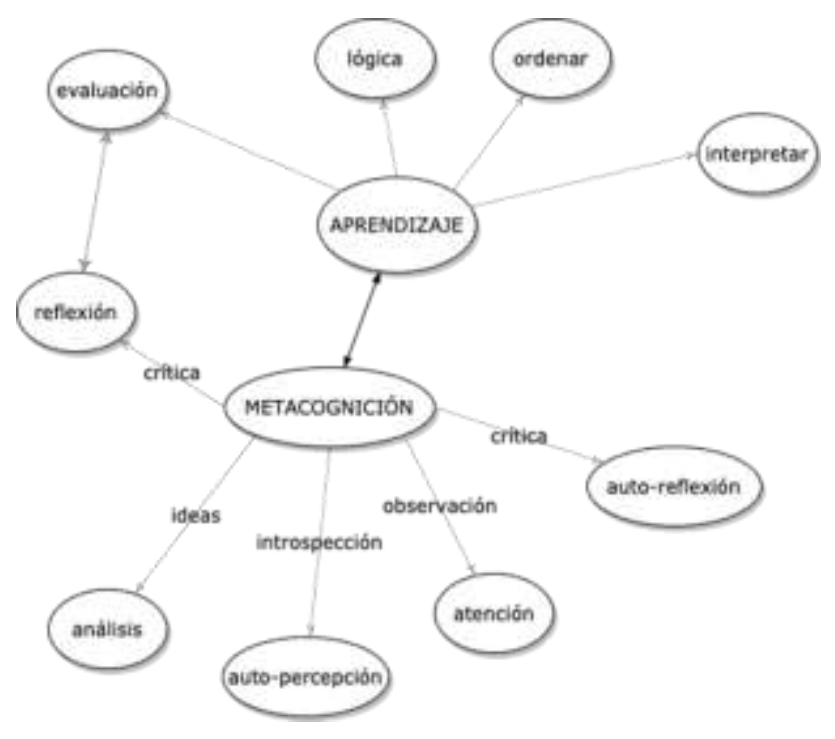

Figura 1 Relación metacognición-aprendizaje

El reflexionar resultó ser un importante componente de la metacognición donde elementos como el análisis, recuerdo, concentración, retrospección, hipótesis lógica, memorización, evaluación y sentido racional, tuvieron cargas factoriales amplias por la mayoría de los sustentantes al considerar los anteriores determinantes en la resolución de problemas.

Si bien dichos componentes hicieron referencia sólo a algunos de los aspectos principales del aprendizaje lógico-matemático, estos brindaron una posible pertinencia en el aprendizaje mediante tareas de tipo reflexivo, las cuales influyeran en una mejor comprensión de los contenidos temáticos, la relación entre estos conforme a las materias en curso, identificar su implicación práctica fuera del contexto escolar y finalmente, propiciar mejores bases para la generación de nuevo conocimiento.
Adicionalmente, los elementos integrados en el instrumento conforme a la reflexión sobre la reflexión, la auto-indagación de la persona; conformaron un bloque que se caracterizó por la inclusión de aspectos cognitivos como la motivación, resolución, y destreza; así como elementos metacognitivos como el análisis, atención, verificación y finalmente elementos de aprendizaje como la lógica, interpretación, evaluación y ordenación.

\section{Conclusiones}

De forma tradicional en el ámbito educativo, la mayoría de este tipo de instituciones en México y demás países, fundamentan el aprendizaje de los alumnos en una estructuración de conocimientos que, conforme organismos federales institucionales consideran los siguientes aspectos: el deber ser y el deber hacer del conocimiento; esta estructura identificada como el currículo representa pues la guía a seguir para formar personas en su dimensión moral, cognitiva y social. Sin embargo, existen gran diversidad de formas, métodos, técnicas y modelos que brindan alternativas para cumplir el cometido concebido de la educación, pero no son estas opciones la panacea del aprendizaje.

La evolución y transformación constante incluyen siempre nuevos paradigmas y necesidades de conocimiento, es por esto que siempre existen nuevas fronteras educativas donde el conocimiento sigue siendo el baluarte principal de todo ser humano.

En el aprendizaje interviene un factor que bien pudiera ser un elemento de cambio tanto en la forma como en el modo de acompañar el proceso educativo, nos referimos a la metacognición. Este concepto que tiene sus orígenes en aportaciones de autores como Piaget y Vygotsky, e introducido por Flavell y representa una más de las alternativas de las cuales podemos disponer para enriquecer el quehacer educativo, es utilizado a la fecha a través de una sencilla tarea que lo define: reflexionar; es evidente que tiene muchas más connotaciones que la reflexión per se, pero lo propuesto en este trabajo de investigación, fue una forma alterna de la metacognición donde se permitiera un acercamiento a la tesis principal aquí propuesta: la potencialización del aprendizaje. 
La metacognición es una tarea inherente en el ser humano y que inicia con los primeros años de su vida donde empieza a darse cuenta de los hechos propios y ajenos del mundo que le rodea; su uso en el sector educativo y específicamente en nuestro país también es ya una realidad, o por lo menos así lo presentan los actuales planes y programas de estudio de la educación básica y superior de nuestro país. Ahora bien, ¿qué situación se presentaría en el ámbito educativo al plantear el uso de procesos metacognitivos frecuentes y constantes en la educación de una persona?, lo anterior no se presenta en ningún sector educativo y es en sí la fórmula que se propone para que a través de la metacognición sea factible la potencialización del conocimiento.

En función del logro de dicha potencialización, se confirmó que una base fundamental del amalgamiento de los ejes aprendizaje y metacognición, es la estructura curricular que se le presenta al alumno donde son clave, los aspectos característicos de todo currículo: guiar la forma de aprender. Para este eje, es coincidente la pertinencia del uso de una estructura curricular con los autores referenciados, así como los paradigmas educativos que al calce se mencionaron.

La única diferencia significativa es la forma en la que se abordaría el uso del currículo al requerir de este un planteamiento orientado en la medida de lo posible a las tareas de reflexión, auto-percepción, evaluación e interpretación de lo que se aprende; por todos los demás elementos operativos que componen este factor, el currículo sería utilizado de la misma forma.

La metacognición es una posible la solución a aprender mejor mediante una mayor reflexión, una presentación diferente de la estructura curricular y un planteamiento más complejo de actividades, pueden ser una alternativa de mejora. Depende de su fomento, que la reflexividad se convierta en el análisis real por parte de la persona que derive en un actuar y pensar diferente, propositivo.
Las estructuras y modelos actuales no necesitan un cambio total, y aunque ese fuera el caso, no se justificaría tampoco dicha acción; por el contrario, es imperativo reconocer la importancia de observar y no simplemente ver, de escuchar y no simplemente oír, de estructurar y no simplemente de memorizar, de interpretar y no solo identificar, tener un mayor grado de autoconciencia que nos permita entender mejor las acciones que realizamos y la forma en la que reaccionamos ante ellas.

Consideremos adicionalmente, que en el ámbito educativo como en cualquier campo, existen cambios constantes pero que, en esencia, la tarea de la educación percibe el mismo fin: generar conocimiento; por lo anterior depende de su fomento, que la reflexividad se convierta en el análisis real por parte del individuo que derive en un actuar y pensar diferente, un actuar propositivo que permita potencializar el aprendizaje.

\section{Referencias}

American Psychological Association. (2010). Manual de Publicaciones de la American Psychologcal Assosiation (Tercera ed.). (M. Guerra Frías, Trad.) México: El Manual Moderno.

Antúnez Pérez, Á., \& León Salazar, Á. (2007). Modelos curriculares en la educación básica venezolana. Revista de Teoría y Didáctica de las Ciencias Sociales.

Arraez, M. (2008). Modelo de integración curricular para la educación básica en Venezuela. Investigación y Postgrado.

Arrieta, M. (2006). La capacidad especial en la educación matemática: estructura y medida. Educación Matemática.

Backhoff Escudero, E., Sánchez Moguel, A., Peón Zapata, M., \& Andrade Muñoz, E. (2010). Comprensión lectora y habilidades matemáticas de estudiantes de educación básica en México: 2000-2005. Revista Electrónica de Investigación Educativa.

Badilla Saxe, E. (2009). Diseño curricular: de la integración a la complejidad. Revista Electrónica Actualidades Investigativas en Educación. 
Bower, G., \& Hilgard, E. (1995). Teorías del Aprendizaje (Segunda ed.). (J. M. Salazár Palacios, Trad.) Trillas.

Cachinero Aviles, A. (2007). Una experiencia de entrenamiento del pensamiento creativo en alumnos de 2do. Ciclo de educación primaria. Psicología Educativa.

Castañeda Quintero, L. (2011). Analizar y entender la enseñanza flexible. Un modelo de análisis de desarrollo curricular. Pixel-Bit. Revista de Medios y Educación.

Clifford, M. M. (1982). Enciclopedia Práctica de la Pedagogía. Oceano.

Cueli, J., de Aguilar, L., Martí, C., Lartigue, T., \& Michaca, P. (1990). Teorías de la Personalidad (Tercera ed.). Trillas.

Díaz Barriga Arceo, F., \& Hernández Rojas, G. (2002). Estrategias Docentes para un Aprendizaje Significativo (Segunda ed.). México: McGraw-Hill.

Díaz Barriga Arceo, F., Lule González, M. d., Pacheco Pinzón, D., Saad Dayán, E., \& RojasDrummond, S. (2011). Metodología de Diseño Curricular para Educación Superior.

Trillas.

Díaz Barriga, Á. (2008). Didáctica y Currículum. Ciudad de México, México: Paidós Educador.

Efklides, A. (2009). The role of metacognitive experiences in the learning process. Psicothema

García, M. I., Tello, F. P., Abad, E. V., \& Moscoso, S. C. (2007). Actitudes, hábitos de estudio y rendimiento en Matemáticas: diferencias por género. Psicothema.

González de Requena Farré, J. A. (2010). Para una reconstrucción genealógica y epistemológica del concepto de metacognición. Revista de Psicología.

Hernández Sampieri, R., Fernández Collado, C., \& Baptista Lucio, P. (2010). Metodología de la Investigación (Quinta ed.). México D.F.: McGraw-Hill.
Klimenko, O., \& Alvares, J. L. (2009). Aprender cómo aprendo: la enseñanza de estrategias metacognitivas. Educación y Educadores.

Nérici, I. G. (1985). Metodología de la Enseñanza (Cuarta ed.). (E. M. Celia, Trad.) Kapelusz Mexicana.

Osses Bustingorry, S., \& Jaramillo Mora, S. (2008). Metacognición: un camino para arender a aprender. Estudios Pedagógicos.

Peñalva Rosales, L. P. (2010). Las matemáticas en el desarrollo de la metacognición. Política y Cultura.

Perkins, D. (2001). La Escuela Inteligente. (V. Gabriela, Trad.) Gedisa.

Peronard Thierry, M. (2009). Metacognición: mente y cerebro. Boletín de filología.

Sáinz, M. C., Flores, V., \& Román, J. M. (2010). Metacognición y competencia de "aprender a aprender" en educación Infantil: una propuesta para facilitar la inclusión. Revista Electrónica Interuniversitaria de Formación del Profesorado.

Silva Córdova, C. (2006). Educación matemática y procesos de metacognitivos en el aprendizaje. Revista del centro de investigación. Universidad La Salle. 